\title{
Biodiversity of fungi on submerged wood in Hong Kong streams
}

\author{
Clement K. M. Tsui*, Kevin D. Hyde, I. John Hodgkiss \\ Centre for Research in Fungal Diversity, Department of Ecology and Biodiversity, The University of Hong Kong, \\ Pokfulam Road, Hong Kong
}

\begin{abstract}
Woody substrates were collected in 5 freshwater streams in Hong Kong and the fungi on these substrates were investigated. A total of 153 species were identified, comprising 61 ascomycete and 92 mitosporic taxa, and $20 \%$ were previously undescribed. About $30 \%$ of the ascomycetes belonged to the genera Annulatascus (Annulatascaceae), Aniptodera, Savoryella (Halosphaeriaceae), Ophioceras, Pseudohalonectria (Magnaporthaceae) and Massarina (Lophiostomataceae). Endophragmiella, Helicosporium and Sporoschisma were common mitosporic genera. Species overlap occurred between different streams, and a few fungi were common in Hong Kong streams. Species composition in the Tung Chung River was distinct, and factors causing variations are discussed. Sampling techniques, the ecological role and distribution of fungi on submerged wood are discussed.
\end{abstract}

KEY WORDS: Ascomycetes · Biodiversity · Ecology · Freshwater - Hyphomycetes · Lignicolous · Stream . Wood

\section{INTRODUCTION}

Dead wood is an essential component of stream ecosystems. Fallen tree trunks, branches and twigs regulate stream dynamics by increasing the retention of organic matter, and provide habitats for a large number of organisms, including fungi, insects and even fish (Triska \& Cromack 1980). Decomposition of these woody substrates is important in nutrient cycling and the rate of wood decay is determined by both physical and biological factors (Harmon et al. 1986). The role of freshwater fungi in the decomposition of organic matter is vital in detritus-based stream systems because nutrients and energy locked up within leaves and wood are released, and these can be used at higher trophic levels (Graça 1993). The conditioned leaves are more palatable to stream insects (Graça 1993).

Freshwater fungi are diverse in taxonomic groups and numbers (Goh \& Hyde 1996). Aquatic hyphomycetes, known as Ingoldian fungi, on wood and leaves have been well investigated with respect to their distribution and biodiversity (Shearer \& Webster 1991, Bär-

\footnotetext{
•E-mail: clementsui@hotmail.com
}

locher 1992, Gönczöl \& Révay 1997), and there are more than 100 species. Research on lignicolous ascomycetes and mitosporic fungi, mainly dematiaceous, has been focused on systematics (Goh et al. 1997a,b, Hyde et al. 1997, Crane et al. 1998). Biodiversity studies of these fungi have been carried out in the United States (Shearer \& von Bodman 1983, Shearer \& Crane 1986, Shearer 1993) and more recently in some tropical and subtropical countries (Hyde \& Goh 1997, 1998a,b, Hyde et al. 1998, Goh \& Hyde 1999). During a single collection of 100 woody substrates 57 species were identified (Goh \& Hyde 1999), and more than 600 species have been described from woody substrates in freshwater ecosystems (M. K. M. Wong et al. 1998).

Inter-stream variations in aquatic hyphomycete communities have been observed globally (Wood-Eggenschwiler \& Bärlocher 1985) and regionally (Chauvet 1991, Raviraja et al. 1998). The fungal communities also exhibited longitudinal and seasonal dynamics (Révay \& Gönczöl 1990, Gönczöl \& Révay 1993, 1997). For the lignicolous ascomycetes and dematiaceous hyphomycetes, little species-overlap occurred between temperate and tropical fungal communities (Shearer 1993, Hyde \& Goh 1998b). Local differences in fungal communities, however, have received less attention. The 
aims of this study were to characterize the biodiversity of wood-inhabiting fungi in different freshwater habitats in Hong Kong, and to investigate if there were variations in species composition in different streams.

\section{METHODS}

Location. Hong Kong is situated on the southern coast of China. It lies between latitudes $22^{\circ} 9^{\prime}$ and $22^{\circ} 37^{\prime} \mathrm{N}$ and longitudes $113^{\circ} 52^{\prime}$ and $114^{\circ} 30^{\prime} \mathrm{E}$, less than $192 \mathrm{~km}$ south of the Tropic of Cancer. Although located in the tropics, Hong Kong has a seasonal climate, and there is a marked seasonal temperature difference. On average, a hot wet monsoon dominates fromin eāiny iviay to the end of September, and is replaced by a cool, dry season from November to March; the seasons are separated by 2 shorter periods of transitional weather (Dudgeon \& Corlett 1994).

Study sites. Five unpolluted streams draining in separate watersheds of the New Territories and Lantau Island, Hong Kong, were selected based on accessibility and lack of human disturbance (Fig. 1). There have been few marked inter-site differences in nutrient status and chemical characteristics in many unpolluted streams (Dudgeon 1989, Dudgeon \& Corlett 1994). Some water parameters of the streams are given in Table 1 (Chan 1997). The waters are soft and slightly acidic. Dissolved oxygen, and $\mathrm{pH}$ of the streams were approximately the same except for their concentration of nitrate and conductivity. They differed with respect to riparian vegetation and shading. Bride's Pool (BP), Hang Cho Shui (HCS), the Lam Tsuen River (LTR) and the Shing Mun Reservoir stream (SM) are surrounded by dense, tall, broad-leaved evergreen trees, and are partially shaded. The Tung Chung River (TCR), however, is largely unshaded, and the riparian vegetation consists mainly of shrubs. Most woody substrates from this stream were collected from one of its pools. The river stream bed of all habitats contains gravel and boulders.

Collection and processing of samples. Fifty woody substrates were randomly collected in BP (January 1999), HCS (September 1998), LTR (December 1996), SM (January 1998 and October 1998) and TCR (August 1997). The samples ranged from twig (ca $1 \mathrm{~cm}$ diameter $\times 30 \mathrm{~cm}$ length) to larger parts of logs $(5 \mathrm{~cm}$ diameter $\times 30 \mathrm{~cm}$ length). All the samples were placed in plastic bags in the field, and returned to the laboratory. They were incubated in plastic boxes $(50 \times 10 \times 10 \mathrm{~cm})$ lined with moistened tissue paper at $26^{\circ} \mathrm{C}$ for $6 \mathrm{wk}$. The woody substrates were examined on Day 7 and then over next $30 \mathrm{~d}$ under a dissecting microscope for fruiting bodies, and any fungi present were recorded, identified and isolated following the method described in Hyde \& Goh (1999).

Data analysis. Numbers of species, frequencies of occurrence of each species and abundance (total occurrence of all fungi) were recorded and calculated for each sampling site. The species diversity of each sampling site was calculated using Shannon-Wiener's index $(H)$ (Begon et al. 1993):

$$
H=-\sum\left(N_{i} / N\right) \log ^{2}\left(N_{i} / N\right)
$$

where $N=$ the number of individuals, and $N_{s}=$ the number of individuals in the $i$ th species. Frequency of occurrence was calculated based on the following formula:

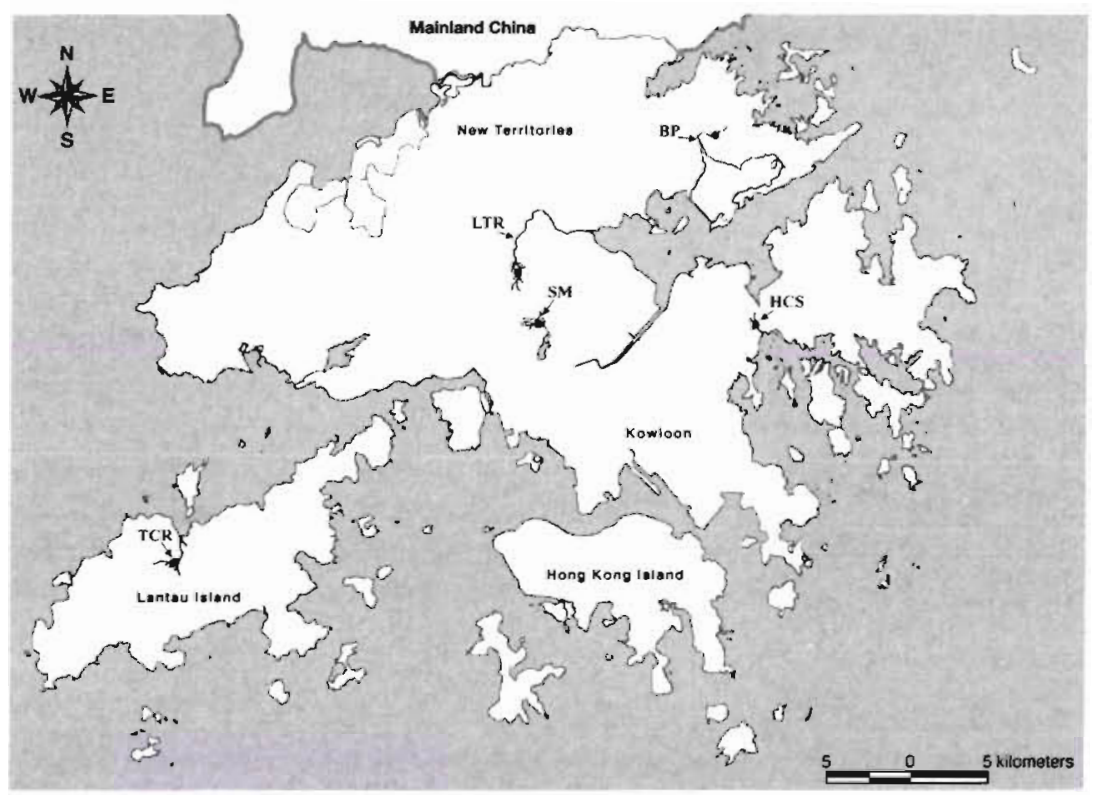

Fig. 1. A map of Hong Kong showing the freshwater sites investigated in this study. BP: Bride's Pool; HCS: Hang Cho Shui; LTR: the Lam Tsuen River; SM: a stream in Shin Mun Reservoir; TCR: the Tung Chung River 
Table 1. Water parameters of all streams (Chan 1997) (BP: Bride's Pool; HCS: Hang Cho Shui; LTR: the Lam Tsuen River; SM: a stream in Shing Mun Reservoir; TCR: the Tung Chung River)

\begin{tabular}{|c|c|c|c|c|c|}
\hline & $\begin{array}{c}\mathrm{BP} \\
22^{\circ} 30^{\prime} \mathrm{N} \\
114^{\circ} 14^{\prime} \mathrm{E}\end{array}$ & $\begin{array}{c}\mathrm{HCS} \\
22^{\circ} 24^{\prime} \mathrm{N}, 114^{\circ} 16^{\prime} \mathrm{E} \\
\text { (data obtained from } \\
\text { a stream nearby) }\end{array}$ & $\begin{array}{c}\text { LTR } \\
22^{\circ} 27^{\prime} \mathrm{N} \\
114^{\circ} 08^{\prime} \mathrm{E}\end{array}$ & $\begin{array}{c}\mathrm{SM} \\
22^{\circ} 24^{\prime} \mathrm{N} \\
114^{\circ} 09^{\prime} \mathrm{E}\end{array}$ & $\begin{array}{c}\text { TCR } \\
22^{\circ} 16^{\prime} \mathrm{N} \\
113^{\circ} 56^{\prime} \mathrm{E}\end{array}$ \\
\hline Dissolved oxygen $\left(\mathrm{mg} \mathrm{l}^{-1}\right)$ & 8.1 & 7.7 & 7.8 & 8 & 9.5 \\
\hline Conductivity $\left(\mu \mathrm{S} \mathrm{\textrm {cm } ^ { - 1 } )}\right.$ & 39.2 & 71.9 & 91.1 & 44.4 & 75.4 \\
\hline $\mathrm{pH}$ & 6.6 & 5.98 & 6.09 & 6.77 & 6.4 \\
\hline Nitrates $\left(\mathrm{mg} \mathrm{l}^{-1}\right)$ & 4.0 & 3 & 7.0 & 4 & 1 \\
\hline Phosphates $\left(\mathrm{mg} \mathrm{l}^{-1}\right)$ & 0 & 0.02 & 0 & 0 & 0 \\
\hline Total suspended solid $\left(\mathrm{mg} \mathrm{l}^{-1}\right)$ & 1 & 2.8 & 4 & 0.8 & 1.8 \\
\hline
\end{tabular}

No. of samples of wood that a particular species occurred on $\times 100$ No. of samples of wood examined

Dominance-diversity curves were plotted as a reflection of the relative abundance of species in each sampling habitat (Kent \& Coker 1992). To compare the similarity of the species composition between different habitats, Sørensen's index of similarity $(S)$ was applied (Magurran 1988). The index was calculated with the formula: $S=2 c / a+b$ where $a=$ total number of species at site $1, b=$ total number of species at site 2 , and $c=$ number of species common to both sites. Similarity is expressed with values between 0 (no similarity) and 1 (absolute similarity).

\section{RESULTS}

\section{Mycological diversity}

A total of 153 fungal taxa were collected (Table 2), consisting of 61 ascomycete (representing $40 \%$ of all mycota) and 92 mitosporic fungi (60\%). About $20 \%$ of the fungi were previously undescribed. Most ascomycete species belonged to the families Annulatascaceae, Halosphaeriaceae, Lasiosphaeriaceae, Lophiostomataceae and Magnaporthaceae (Barr 1990. Hawksworth et al. 1995, S. W. Wong et al. 1998, sen- su Shearer et al. 1999) (Fig. 2). Several genera were well represented in aquatic environments and included Aquaticola, Annulatascus, Aniptodera, Halosarpheia, Massarina, and Savoryella (Table 2). Fifty-six mitosporic fungal genera were identified in this study, and Dactylaria and Helicosporium were the most common ones, with 5 species in each genus. Other common genera included Helicomyces, Chloridium, Monodictys and Sporoschisma, each of which had 4 species (Table 2).

The following species occurred frequently in more than 1 stream: Aniptodera chesapeakensis, Aquaticola rhomboidea, Candelabrum brocchiatum, Helicomyces torquatus, Helicosporium sp., Massarina ingoldiana, Massarina thalassioidea, Savoryella lignicola, Sporoschisma nigroseptatum, Sporochisma uniseptatum (Table 3). Some species (Table 2), although Iow in frequency of occurrence, were also recorded in all streams, e.g. Annulatascus velatisporus, Massarina bipolaris, Ophioceras dolichostomum, Torrentispora fibrosa, Acrogenospora sphaerocephala, Ellisembia opaca, Exserticlava vasiformis, Sporidesmiella hyalospermum var. hyalospermum, and Sporoschisma saccardoi. Some species were, however, more abundant in a particular stream, such as Phaeoisaria clematidis in HCS, Stanjehughesia nigroaca and Monotosporella setosa var, macrocarpa in the TCR, and Massarina purpurascens in the LTR.

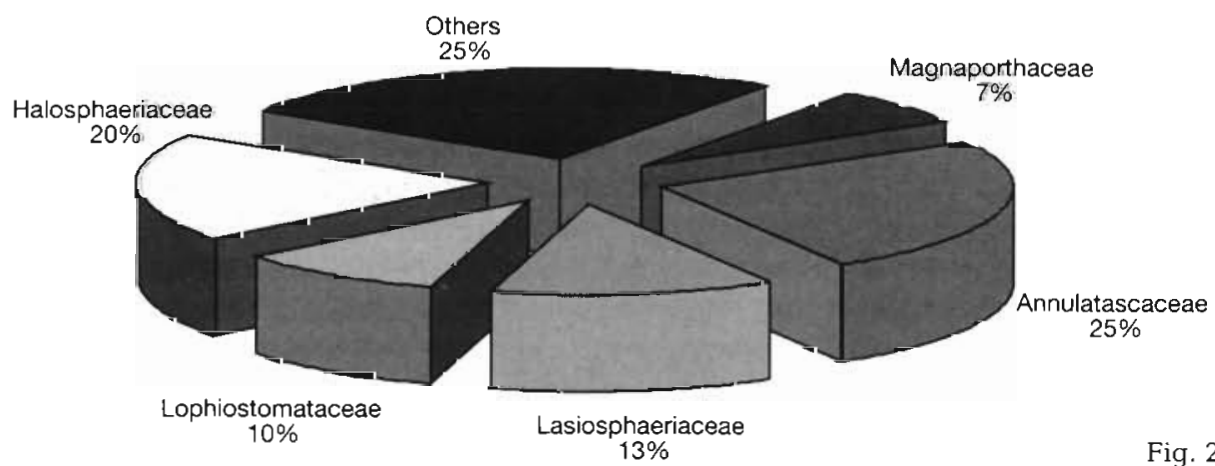

Fig. 2. Ascomycete families encountered 
Table 2. Frequency of occurrence of fungi on submerged wood in streams in Hong Kong

\begin{tabular}{|c|c|c|c|c|c|c|}
\hline Species name & $\mathrm{BP}$ & $\mathrm{HCS}$ & LTR & SM1 & $\mathrm{SM} 2$ & TCR \\
\hline \multicolumn{7}{|l|}{ Ascomycetes } \\
\hline \multicolumn{7}{|l|}{ Annulatascaceae } \\
\hline Annulatascus biatrisporus K. D. Hyde & - & - & - & - & $2 \%$ & - \\
\hline Annulatascus sp. & - & - & $2 \%$ & - & - & - \\
\hline Annulatascus velatisporus K. D. Hyde & $6 \%$ & - & - & $4 \%$ & $8 \%$ & $2 \%$ \\
\hline Aquaticola hyalomura W. H. Ho, K. M. Tsui, K. D. Hyde \& Hodgkiss & $2 \%$ & $2 \%$ & $6 \%$ & $6 \%$ & - & - \\
\hline Aquaticola sp. & - & $2 \%$ & - & - & $2 \%$ & $2 \%$ \\
\hline Aquaticola sp. & $\sim$ & - & - & - & $2 \%$ & - \\
\hline Aquaticola rhomboidea W. H. Ho, K. M. Tsui, K. D. Hyde \& Hodgkiss & $4 \%$ & $10 \%$ & $18 \%$ & $12 \%$ & $8 \%$ & - \\
\hline Ascotaiwania hsilio H. S. Chang \& S.-Y. Hsieh & - & $2 \%$ & - & - & - & - \\
\hline Cateractispora viscosa S. W. Wong & - & $2 \%$ & - & - & - & - \\
\hline Clohiesia corticola K. D. Hyde & - & $2 \%$ & - & - & - & - \\
\hline Clohiesia lignicola K. M. Tsui, K. D. Hyde \& Hodgkiss & - & - & - & $2 \%$ & - & $6 \%$ \\
\hline Pseudoproboscispora aquatica S. W. Wong \& K. D. Hyde & $2 \%$ & - & $6 \%$ & $4 \%$ & - & - \\
\hline Rivulicola incrustata K. D. Hyde & $4 \%$ & - & - & - & - & - \\
\hline Torrentispora fibrosa K. D. Hyde & - & - & $2 \%$ & $4 \%$ & $2 \%$ & $2 \%$ \\
\hline \multicolumn{7}{|l|}{ Halosphaeriaceae } \\
\hline Aniptodera chesapeakensis Shearer & $6 \%$ & $2 \%$ & $16 \%$ & $30 \%$ & $18 \%$ & $4 \%$ \\
\hline Aniptodera inflatiascigera K. M. Tsui, K. D. Hyde \& Hodgkiss & - & - & $2 \%$ & - & - & - \\
\hline Aniptodera lignatilis K. D. Hyde & - & - & $2 \%$ & - & - & - \\
\hline Aniptodera sp. & - & - & - & - & - & $2 \%$ \\
\hline Halosarpheia aquadulcis Hsieh, H. S. Chang \& E. B. G. Jones & $2 \%$ & - & - & - & - & - \\
\hline Halosarpheia heteroguttulatta S. W. Wong \& K. D. Hyde & - & - & $2 \%$ & $2 \%$ & - & - \\
\hline Halosarpheia lotica Shearer & - & - & $4 \%$ & - & - & - \\
\hline Lutrellia estuarina Shearer & - & - & - & - & - & $4 \%$ \\
\hline Nais aquatica K. D. Hyde & - & - & $4 \%$ & - & - & - \\
\hline Savoryella aquatica K. D. Hyde & - & - & - & $4 \%$ & - & - \\
\hline Savoryella lignicola E. B. G. Jones \& R. A. Eaton & $26 \%$ & - & - & $10 \%$ & $4 \%$ & - \\
\hline Savoryella verrucosa Minoura \& Muroi & - & - & - & $2 \%$ & - & - \\
\hline \multicolumn{7}{|l|}{ Clypeosphariaceae } \\
\hline Jobellisia sp. & - & - & - & - & - & $2 \%$ \\
\hline \multicolumn{7}{|l|}{ Lasiosphaeriaceae } \\
\hline Cercophora costariensis (Carroll \& Munk) O. Hilber \& R. Hilber & - & - & - & $4 \%$ & - & - \\
\hline Cercophora caudata (Curr.) Lundq. & - & - & $8 \%$ & - & - & - \\
\hline Chaetosphaeria hiugensis Hino & - & - & - & $2 \%$ & - & - \\
\hline Lasiosphaeria sp. & - & - & - & - & $2 \%$ & - \\
\hline Lasiosphaeria immersa P. Karst & - & - & $2 \%$ & - & - & - \\
\hline Lasiosphaeria lapaziana Carroll \& Munk & - & $4 \%$ & - & - & - & - \\
\hline Melanochaeta hemipsila (Berk \& Broome) E. Mull., Harr. \& Sulmont & - & - & - & $4 \%$ & - & - \\
\hline Melanochaeta sp. & - & - & - & - & - & $2 \%$ \\
\hline \multicolumn{7}{|l|}{ Lophiostomataceae } \\
\hline Massarina corticola (Fuckel) L. Holm & $2 \%$ & - & - & - & - & - \\
\hline Massarina bipolaris K. D. Hyde & - & $6 \%$ & $2 \%$ & $2 \%$ & $6 \%$ & $6 \%$ \\
\hline Massarina ingoldiana Shearer \& K. D. Hyde & $8 \%$ & - & $8 \%$ & $10 \%$ & $12 \%$ & - \\
\hline Massarina purpurascens K. D. Hyde \& Aptroot & $2 \%$ & - & $14 \%$ & $2 \%$ & - & - \\
\hline Massanina thalassioidea K. D. Hyde \& Aptroot & $10 \%$ & $6 \%$ & $16 \%$ & $6 \%$ & $12 \%$ & - \\
\hline Vaginatispora aquatica K. D. Hyde & $4 \%$ & - & - & - & - & - \\
\hline \multicolumn{7}{|l|}{ Hypocreaceae } \\
\hline Nectria haematoccoca Berk. \& Br. & - & $2 \%$ & $6 \%$ & $2 \%$ & - & - \\
\hline \multicolumn{7}{|l|}{ Magnaporthaceae } \\
\hline Ophioceras commune Shearer & - & $4 \%$ & $16 \%$ & - & - & - \\
\hline Ophioceras dolichostomum (Berk. \& Curk. ) Sacc. & $4 \%$ & - & $8 \%$ & $4 \%$ & $6 \%$ & - \\
\hline Pseudohalonectria adversaria Shearer & - & - & - & $4 \%$ & - & - \\
\hline Pseudohalonectria lignicola Minoura \& Muroi & - & - & - & $2 \%$ & - & - \\
\hline \multicolumn{7}{|l|}{ Massariaceae } \\
\hline Caryospora minima Jeffers & $2 \%$ & - & - & - & - & - \\
\hline
\end{tabular}


Table 2. (continued)

\begin{tabular}{|c|c|c|c|c|c|c|}
\hline Species name & $\mathrm{BP}$ & $\mathrm{HCS}$ & LTR & SM1 & $\mathrm{SM} 2$ & TCR \\
\hline \multicolumn{7}{|l|}{ Orbiliaceae } \\
\hline Orbilia luterobella (Nyl.) P. Karst. & - & - & $2 \%$ & $2 \%$ & - & - \\
\hline \multicolumn{7}{|l|}{ Phyllachoraceae } \\
\hline Glomerella sp. & $2 \%$ & - & - & - & - & $2 \%$ \\
\hline \multicolumn{7}{|l|}{ Platystomaceae } \\
\hline Astrosphaeriella trochus (Penz. \& Sacc) D. Hawksw. & - & - & - & $2 \%$ & - & - \\
\hline \multicolumn{7}{|l|}{ Pleomassariaceae } \\
\hline Kirschsteiniothelia elasterascus Shearer & - & - & $2 \%$ & - & - & - \\
\hline \multicolumn{7}{|l|}{ Valsaceae } \\
\hline Diaporthe sp. & - & $6 \%$ & - & - & - & - \\
\hline \multicolumn{7}{|l|}{ Incertae sedis } \\
\hline Jahnula bipolaris (K. D. Hyde) K. D. Hyde & - & - & - & $4 \%$ & $8 \%$ & - \\
\hline Phomatospora berkeleyi Sacc. & $2 \%$ & - & - & - & $4 \%$ & - \\
\hline Saccardoella aquatica K. M. Tsui, K. D. Hyde, Hodgkiss \& Goh & - & - & $2 \%$ & - & - & - \\
\hline Ascomycete sp. 1 & - & - & $2 \%$ & - & - & - \\
\hline Ascomycete sp. 2 & - & - & - & - & - & $2 \%$ \\
\hline Ascomycete sp. 3 & - & $2 \%$ & - & - & - & - \\
\hline Ascomycete sp. 4 & - & - & - & - & - & $2 \%$ \\
\hline Ascomycete sp. 5 & $4 \%$ & - & - & - & - & - \\
\hline Ascomycete sp. 6 & - & - & - & $4 \%$ & - & - \\
\hline \multicolumn{7}{|l|}{ Coelomycetes } \\
\hline Coelomycetes sp. 1 & $6 \%$ & - & $2 \%$ & - & - & - \\
\hline Coelomycetes sp. 2 & - & $2 \%$ & $2 \%$ & - & - & - \\
\hline Coelomycetes sp. 3 & - & - & $6 \%$ & - & - & - \\
\hline Satchmopsis sp. & - & - & $4 \%$ & - & - & - \\
\hline Trematophora sp. & - & - & $2 \%$ & - & - & - \\
\hline \multicolumn{7}{|l|}{ Hyphomycetes } \\
\hline Acrogenospora sphaerocephala (Berk. \& Broome) M. B. Ellis & - & $2 \%$ & - & $2 \%$ & $4 \%$ & $4 \%$ \\
\hline Aegerita sp. & - & - & $2 \%$ & - & - & - \\
\hline Anguillospora gigantea Ranzoni & - & - & $6 \%$ & - & - & - \\
\hline Arachnophora hughesii R. F. Castaneda \& Guarro & - & - & $6 \%$ & - & - & - \\
\hline Bahusutrabeeja dwaya Subram. \& Bhatt & - & - & $2 \%$ & - & - & - \\
\hline Berkleasmium concinnum (Berk.) Moore & - & - & - & - & - & $4 \%$ \\
\hline Berkleasmium corticola (Karst.) Moore & - & $2 \%$ & - & - & - & - \\
\hline Brachiosphaera tropicalis Nawawi & - & - & $4 \%$ & - & - & - \\
\hline Cacumisporium sp. & - & - & - & - & - & $4 \%$ \\
\hline Camposporium antennatum Harkn. & - & - & $12 \%$ & $2 \%$ & - & - \\
\hline Canalisporium caribense (Hol.-Jech. \& Mercado) Nawawi \& Kuthub. & - & $2 \%$ & $2 \%$ & - & $2 \%$ & - \\
\hline Canalisporium pulchrum (Hol.-Jech. \& Mercado) Nawawi \& Kuthub. & - & - & $2 \%$ & - & $2 \%$ & - \\
\hline Cancellidium applanatum Tubaki & - & - & - & $4 \%$ & $2 \%$ & - \\
\hline Candelabrum brocchiatum Tubaki & $16 \%$ & $8 \%$ & $8 \%$ & $24 \%$ & $12 \%$ & - \\
\hline Candelabrum sp. & - & - & - & $10 \%$ & - & - \\
\hline Chaetochalara sp. & $2 \%$ & $2 \%$ & - & - & $2 \%$ & - \\
\hline \multicolumn{7}{|l|}{ Chloridium botryoideum (Corda) Hughes var. } \\
\hline minutum (Sacc) W. Gams \& Hol.-Jech. & - & - & - & - & - & $6 \%$ \\
\hline Chloridium matsushmae W. Gams \& Hol.-Jech. & - & $2 \%$ & - & - & - & - \\
\hline Chloridium sp. & - & - & - & $2 \%$ & - & - \\
\hline Cladorrhinum foecundissimium Sacc. \& Marchal & - & - & $2 \%$ & $4 \%$ & - & - \\
\hline Codineae britannica Ellis & $2 \%$ & - & - & - & - & - \\
\hline Cordana musae (Zimm.) Höhnel & - & - & - & - & - & $2 \%$ \\
\hline Dactylaria sp. & - & - & - & - & - & $2 \%$ \\
\hline Dactylaria hoogi R. F. Castaneda \& W. B. Kendr. & - & - & $6 \%$ & - & - & - \\
\hline Dactylaria hyalotunicata K. M. Tsui, Goh \& K. D. Hyde & $2 \%$ & - & $2 \%$ & $6 \%$ & - & $4 \%$ \\
\hline Dactylaria malaysianum Matsushi. & - & - & - & - & - & $4 \%$ \\
\hline Dactylaria triseptata (Matsush.) R. F. Castaneda \& W. B. Kendr. & $2 \%$ & - & $2 \%$ & - & - & $2 \%$ \\
\hline
\end{tabular}


Table 2. (continued)

\begin{tabular}{|c|c|c|c|c|c|c|}
\hline Species name & $\mathrm{BP}$ & $\mathrm{HCS}$ & LTR & SM1 & SM2 & TCR \\
\hline Dictyochaeta coffeae (Maggi \& Persiani) Kuthubu. \& Nawawi & $2 \%$ & - & $12 \%$ & - & - & - \\
\hline Dictyochaeta subfuscospora Kuthub. \& Nawawi & - & - & - & - & - & $2 \%$ \\
\hline Dictyosporium digitatum J. L. Chen, C. H. Hwang \& S. S. Tzean & - & - & - & $2 \%$ & - & - \\
\hline Elegantimyces sporidesmiopsis Goh, K. M. Tsui \& K. D. Hyde & - & - & $2 \%$ & - & - & - \\
\hline Ellisembia leonense McKenzie & - & - & $4 \%$ & - & - & - \\
\hline Ellisembia opaca (Cooke \& Harkn.) Subram. & $2 \%$ & $8 \%$ & $4 \%$ & $6 \%$ & - & $2 \%$ \\
\hline Endophragmiella sp. 1 & - & - & $2 \%$ & - & - & - \\
\hline Endophragmiella sp. 2 & - & - & - & $2 \%$ & - & - \\
\hline Exserticlava vasiformis (Matsush) S. Hughes & - & - & - & $8 \%$ & - & - \\
\hline Gonytrichum chlamydosporium var. chlamydospoium & - & $2 \%$ & $2 \%$ & $2 \%$ & - & $2 \%$ \\
\hline Haplochalara angulospora Linder & - & $6 \%$ & - & - & - & - \\
\hline Helicomyces roseus Link & - & $8 \%$ & $6 \%$ & $2 \%$ & $4 \%$ & - \\
\hline Helicomyces torquatus Lane \& Shearer & $14 \%$ & - & - & - & $10 \%$ & - \\
\hline Helicosporium aquaticus sp. nov. & $2 \%$ & - & - & - & $4 \%$ & - \\
\hline Helicosporium griseum Berkeley \& Curtis & $18 \%$ & $12 \%$ & $14 \%$ & $18 \%$ & - & - \\
\hline Helicosporium guianenses Linder & - & $2 \%$ & - & $2 \%$ & - & - \\
\hline Helicosporium pallidum Cesati & $10 \%$ & - & $16 \%$ & $4 \%$ & $2 \%$ & $4 \%$ \\
\hline Helicosporium sp. & $2 \%$ & - & - & - & - & - \\
\hline Isthmolongispora intermedia Matsush. & - & - & - & - & - & $4 \%$ \\
\hline Janetia cuviapicis Goh \& K. D. Hyde & - & $2 \%$ & - & - & - & $6 \%$ \\
\hline Melanocephala cupulifera S. Hughes & - & $4 \%$ & - & - & - & - \\
\hline Menisporopsis multisetulata K. M. Tsui, Goh \& K. D. Hyde & - & - & $4 \%$ & - & - & - \\
\hline Monodictys sessilis Hol.-Jech. & - & $2 \%$ & - & - & - & - \\
\hline Monodictys putredinis (Wallr.) S. Hughes & - & $2 \%$ & $2 \%$ & - & - & - \\
\hline Monodictys striata (Petch) V. Rao \& de Hoog & - & $2 \%$ & - & - & - & - \\
\hline Monodictys sp. & - & $8 \%$ & $2 \%$ & - & $4 \%$ & - \\
\hline Monotosporella setosa var. macrocarpa S. Hughes & $2 \%$ & - & - & - & - & $10 \%$ \\
\hline Monotosporella sp. & - & $6 \%$ & - & - & - & - \\
\hline Neta lignicola Shearer & - & - & - & $2 \%$ & - & - \\
\hline Phaeoisaria clematidis (Fuckel) S. Hughes & $4 \%$ & $12 \%$ & $10 \%$ & $8 \%$ & - & - \\
\hline Phialogeniculata guadelcanalensis Matsush. & - & - & - & $2 \%$ & - & $2 \%$ \\
\hline Pleurothecium recurvatum (Morgan) Höhnel & $2 \%$ & $2 \%$ & - & $2 \%$ & $2 \%$ & - \\
\hline Pseudobotrytis terrestis (Timonin) Subram. & - & $4 \%$ & - & - & - & - \\
\hline Rhinocladiella sp. & $2 \%$ & $2 \%$ & $6 \%$ & - & - & - \\
\hline Sarcopodium circinatum Ehrenb ex Schlecht & - & - & $2 \%$ & - & - & - \\
\hline Spadicoides atra (Corda) Hughes & - & - & - & - & - & $2 \%$ \\
\hline Spadicoides abovatum (Cooke \& Ellis) S. Hughes & - & - & - & - & - & $2 \%$ \\
\hline Spirosphaera floriformis Beverwijk & - & - & - & $8 \%$ & $4 \%$ & $4 \%$ \\
\hline Sporidesmiella hyalosperum (Corda) P. M. Kirk var. hyalosperum & $2 \%$ & $2 \%$ & $4 \%$ & $2 \%$ & - & - \\
\hline Sporidesmiella incrassata Kuthub. \& Nawawi & - & - & - & - & - & $2 \%$ \\
\hline Sporidesmium macrurum M. B. Ellis & - & - & - & - & - & $2 \%$ \\
\hline Sporidesmium dissolvens Hol.-Jech., Mercado \& Mena & - & - & $2 \%$ & - & - & - \\
\hline Sporidesmium sp. & - & - & - & - & - & $2 \%$ \\
\hline Sporoschisma juvenile Boud. & - & - & $2 \%$ & - & - & - \\
\hline Sporoschisma nigroseptatum D. Rao \& R. Rao & $2 \%$ & $2 \%$ & $10 \%$ & $12 \%$ & $16 \%$ & $12 \%$ \\
\hline Sporoschisma saccardoi E. W. Mason \& S. Hughes & - & $2 \%$ & $4 \%$ & $8 \%$ & $4 \%$ & - \\
\hline Sporoschisma uniseptatum Bhat \& W. B. Kendr. & $12 \%$ & $10 \%$ & $24 \%$ & $8 \%$ & $8 \%$ & - \\
\hline Stanjehughesia nigroaca (B. C. Sutton) Subram. & - & - & - & - & - & $10 \%$ \\
\hline Thozetella effusa B. Sutton \& G. T. Cole & $2 \%$ & - & - & - & - & - \\
\hline Thozetella nivea (Berk. \& F. Mull.) O. Kuntze & - & - & - & - & - & $2 \%$ \\
\hline Tricladium attenuatum Iqbal & $2 \%$ & - & $6 \%$ & $2 \%$ & - & - \\
\hline Triposporium elegans Corda & - & - & - & - & $2 \%$ & - \\
\hline Vanakripa sp. & $2 \%$ & $2 \%$ & - & - & - & - \\
\hline Verticillium $\mathrm{sp}$ & - & - & $4 \%$ & - & $2 \%$ & $6 \%$ \\
\hline Virgariella atra S. Hughes & - & $2 \%$ & & - & - & - \\
\hline Xylomyces elegans Goh, W. H. Ho, K. D. Hyde \& K. M. Tsui & $2 \%$ & - & - & - & $4 \%$ & - \\
\hline Yinmingella mitriformis Goh, K. M. Tsui \& K. D. Hyde & - & - & - & - & - & $2 \%$ \\
\hline Zanclospora brevispora S. Hughes \& W. B. Kendr. var. brevispora & - & - & - & - & - & $2 \%$ \\
\hline Hyphomycetes sp. 1 & - & - & - & - & $4 \%$ & - \\
\hline Hyphomycetes sp. 2 & - & - & - & - & $2 \%$ & - \\
\hline
\end{tabular}




\section{Species richness and abundance}

The highest species diversity was recorded in the LTR in terms of taxa and abundance, followed by the SM (in January 1998). Diversity for the SM, however, dropped remarkably after 9 mo (October 1998). The number of taxa collected in BP, HCS and the TCR was similar. In general, species diversity (Shannon) was high, with values more than 5 for all streams, except BP and SM (October 1998) (Table 4). Dominancediversity curves (Figs. 3 and 4 ) indicate that a high proportion of species occurred once or twice.

The similarity values among all sampling sites are presented in Table 5. The highest similarity in species composition (0.467) was found between the ITR and the SM (January 1998), and this was even higher than the similarity of the same habitat $(0.444)$ sampled in 2 different months in the SM (January and October

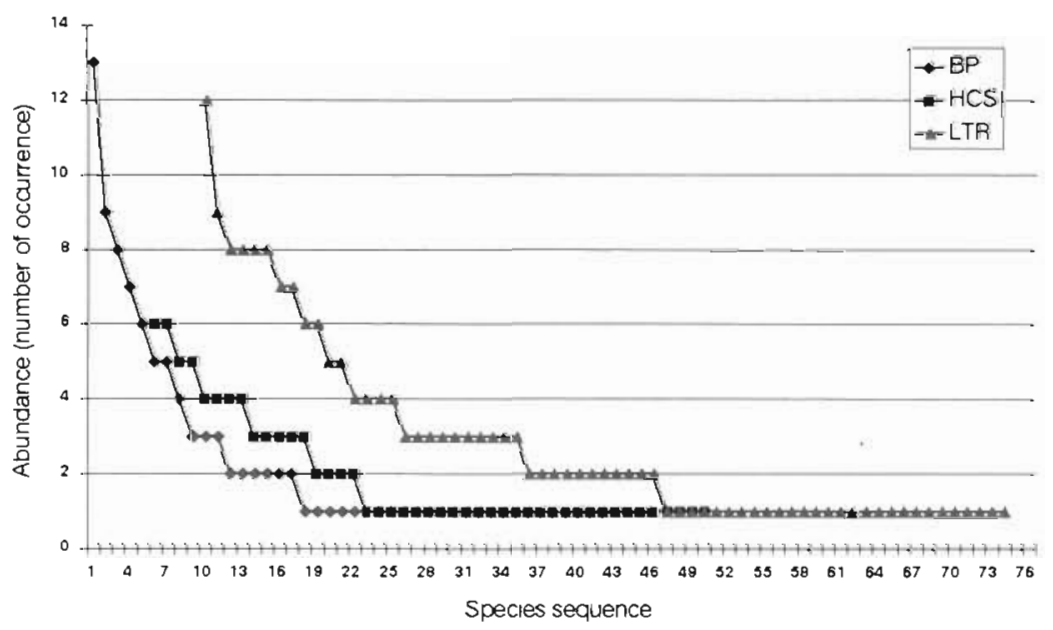

Fig. 3. Dominance-diversity curve for BP, HCS and LTR

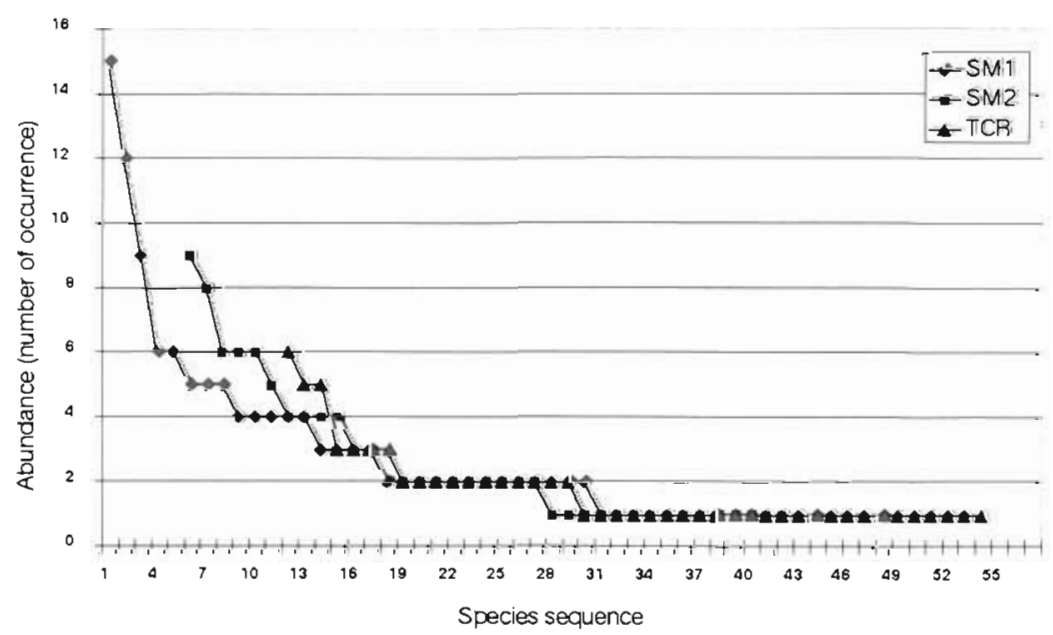

Fig. 4. Dominance-diversity curve for SM1, SM2 and TCR
1998). The TCR, situated on Lantau Island, had low values of similarity when compared with other habitats, implying its fungal community was distinct.

\section{DISCUSSION}

\section{Local variations}

A comparison of the fungal communities at the different streams examined illustrates dissimilarities in species composition. The fungal community at the TCR was particularly distinctive. According to the river continuum concept ( $\mathrm{RCC}$ ), the stream morphology, hydrology, and allochthonous and autochthonous inputs interact to influence the aquatic plankton and invertebrate community (Vannote et al. 1980). Differences in the extent of shading by riparian vegetation affects the amount of detritus in the streams, thus affecting macroinvertebrate community structure (Dudgeon 1989, Dudgeon \& Corlett 1994). BP, HCS, the LTR and SM are largely shaded sites, where the canopies of riparian trees interlock over the streams. These sites have a highly allochthonous food base. On the other hand TCR is surrounded by shrubs and is largely unshaded (Dudgeon \& Corlett 1994). The influence of autochthonous input on the energy base in the TCR may affect the distinct taxonomic composition. The distinct fungal community at the TCR may also be influenced by the fact that woody substrates were mostly collected in a pool (static water) where there was a lower concentration of dissolved oxygen (Ho 1998), in comparison to other lotic (running water) habitats.

In this study, some lignicolous fungal species appeared to be associated with specific woody substrates (having specific color and texture), even though the host species cannot be identified (C.K.M.T. pers. obs.). Perhaps these fungi occurred at different stages during the wood decay process (Ho 1998), but substrate specificity cannot be ruled out. It is well established that changes in aquatic hyphomycete communities are correlated with changes in the riparian vegetation (Thomas et al. 1989, Fabre 1996). Different substrates in streams may selectively inhibit or stimulate colonization of some fungal 
species (Bärlocher 1992) and cause variations in community structure (Thomas et al. 1992). Although 2 studies have shown that the lignicolous fungi have little substratum specificity (Révay \& Gönczöl 1990, Ho 1998), few freshwater ascomycetes occur on both woody and herbaceous substrates (Shearer 1999), which may be an indication of showing substratum specificity.

However, the results were cautiously interpreted further because there appears to be minor differences in water parameters of the streams. Collections at different times provide very different resuits. There is a marked change in fungal communities in the SM in January and October 1998. The quality (length and diameter) of woody substrates was also not considered. The number of basidiomycetes on decaying wood in the terrestrial environment was correlated with the diameter and length of logs (Hoiland \& Bendiksen 1997).
Table 3. Eight most frequently occurring species in each of 6 Hong Kong streams

\begin{tabular}{|lccccccc|}
\hline & BP & HCS & LTR & SM1 & SM2 & TCR \\
\hline Candelabrum brocchiatum & $\times$ & $\times$ & $\times$ & $\times$ & $\times$ & \\
Helicomyces torquatus & $\times$ & & $\times$ & & $\times$ & \\
Helicosporium griseum & $\times$ & $\times$ & & $\times$ & & \\
Helicosporium pallidum & $\times$ & & & & & $\times$ \\
Massarina ingoldiana & $\times$ & & & $\times$ & $\times$ & \\
Massarina purpurascens & $\times$ & & $\times$ & & & \\
Savoryella lignicola & $\times$ & & & $\times$ & & \\
Sporoschisma uniseptatum & $\times$ & $\times$ & $\times$ & & & \\
Massarina thalassioidea & & $\times$ & $\times$ & & $\times$ & \\
Monodictys nigrocephala & & $\times$ & & & & \\
Phaeiosaria clemiatids & & $\times$ & & & & \\
Aquaticola rhomboidea & & $\times$ & $\times$ & $\times$ & $\times$ & \\
Helicomyces roseus & & $\times$ & & & & \\
Aniptodera chesapeakensis & & & $\times$ & $\times$ & $\times$ & \\
Annulatascus velatisporus & & & & & $\times$ & \\
Ophioceras commune & & & $\times$ & & & \\
Candelabrum sp. & & & & $\times$ & & \\
Sporoschisma nigroseptatum & & & & $\times$ & $\times$ & $\times$ \\
Clohiesia lignicola & & & & & & $\times$ \\
Janetia curviapicis & & & & & & $\times$ \\
Monotosporella setosa var. macrocarpa & & & & & & $\times$ \\
Chloridium botryoideum var. minutum & & & & & & $\times$ \\
Stanjehughesia nigroaca & & & & & & $\times$ \\
Verticillium sp. & & & & & & \\
\hline
\end{tabular}

\section{Ecology and distribution}

There are great differences in species composition between Hong Kong and elsewhere, e.g. Australia, Britain, Brunei, Malaysia and South Africa (Hyde \& Goh 1997, 1998a,b, 1999, Ho 1998, Hyde et al. 1998). With the exception of Annulatascus velatisporus, Massarina bipolaris, Savoryella lignicola and Sporoschisma uniseptatum, most common fungi in the freshwater sites were different. Even though there is no comprehensive review dealing with the biogeography of wood-inhabiting fungi in freshwater habitats, temperature and geographical factors are regarded as crucial in determining global distribution patterns (WoodEggenschwiler \& Bärlocher 1985). Only 1 species recorded in the River Coln, UK, has also been found in other tropical or subtropical collections (Hyde \& Goh 1999). Yuen et al. (1999) found that tropical freshwater fungi had faster growth rates than temperate species, although both had similar optimum temperatures for growth. The temperate fungi may be out-competed by tropical fungi in tropical regions. Also, temperature requirements and the capabilities of fungi for producing enzymes varied depending on where they had been isolated from. Fungi may, therefore, have geographical races (Yuen et al. 1999), and this may account for the absence of species in certain regions.

The common ascomycete genera in Hong Kong were Annulatascus, Aniptodera, Halosarpheia, Massarina and Ophioceras. This was consistent with observations from Hyde et al. (1997) that they are common tropical genera. Of the 288 ascomycetes listed by Shearer (1993), only 8 were found in this study, which was an illustration of the difference in fungal communities in tropical and temperate regions.

The common ascomycetes and mitosporic fungi in this study have also been found in the Tai Po Kau stream and Plover Cove Reservoir, Hong Kong (Ho 1998, Goh \& Hyde 1999). It would appear that fungal communities in Hong Kong comprise a 'core group' of a few species, such as Aniptodera chesapeakensis, Annulatascus velatisporus, Aquaticola rhomboidea,

Table 4. Species diversity in various streams. $R$ : Ratio of hyphomycetes to ascomycetes

\begin{tabular}{|lcccccc|}
\cline { 1 - 5 } & BP & HCS & LTR & SM1 & SM2 & TCR \\
\cline { 1 - 5 } Hyphomycetes & 25 & 30 & 36 & 27 & 21 & 29 \\
Ascomycetes & 16 & 14 & 24 & 26 & 16 & 13 \\
Coelomycetes & 1 & 1 & 5 & 0 & 0 & 0 \\
$R$ & 1.47 & 2.14 & 1.58 & 1.04 & 1.31 & 2.23 \\
Number of species & 42 & 45 & 65 & 53 & 37 & 42 \\
Abundance & 103 & 88 & 181 & 144 & 96 & 75 \\
Number of species per sample & 2.1 & 1.8 & 3.8 & 2.9 & 1.9 & 1.5 \\
Shannon diversity index & 4.8 & 5.11 & 5.53 & 5.22 & 4.79 & 5.13 \\
\hline
\end{tabular}


Candelabrum brocchiatum, Helicomyces torquatus, Helicomyces roseus, Helicosporium sp., Massarina ingoldiana, Massarina thalassioidea, Ophioceras dolichostomum, Sporoschisma nigroseptatum, and Sporochisma uniseptatum. M. K. M. Wong et al. (1998) suggested that 'keystone species' occurred in freshwater ecosystems. Since no ascomycetes and mitosporic fungi are likely to be able to produce all of the lignocellulose-degrading enzymes to degrade wood, they may work as a consortium in wood degradation in the freshwater environment, and therefore are vital to the maintenance of the ecosystem (M. K. M. Wong et al. 1998). If a fungus cannot produce lignocellulosedegrading enzymes, it is unlikely to contribute to wood decay or be part of a consortium. In fact there are many microfungi that occur in wood in the terrestrial environment that rely on readily assimilable nutrients and do not decay the wood (Rayner \& Boddy 1988). Further physiological, anatomical and ecological studies are therefore necessary in order to verify if the core group has the ability to form soft rot cavities and to decay wood, and are keystone species in the freshwater ecosystem.

As far as the minimum sampling effort (number of woody substrates) is concerned, 50 could be an optimum number. In this study, the number of taxa identified from single collections at 5 streams in Hong Kong ranged from 37 to 67 . Ho (1998) also collected 71, 79 and 53 fungal taxa from Brunei, Hong Kong (the Tai Po Kau stream) and Malaysia respectively after examining 50 woody substrates. The studies carried out by Hyde and co-workers were based on collections of 100 or more woody substrates. Thirty-nine and 42 taxa were identified from Lake Barrine and Mount Lewis, Australia (Hyde \& Goh 1997, 1998a), and 58 were recorded from the Palmiet River, South Africa (Hyde et al. 1998). Twenty-eight and 34 taxa were recorded from the River Coln, Britain, and from the Seychelles respectively (Hyde \& Goh 1998b, 1999). The differences in species diversity may be related to temperature and geography as discussed above, but questions on the period of incubation and the importance of extensive and in-depth investigation are also raised. Prolonged incubation generally increases the diversity of

Table 5. Index of similarity among different streams

\begin{tabular}{|c|c|c|c|c|c|c|}
\hline & $\mathrm{BP}$ & $\mathrm{HCS}$ & LTR & SM1 & $\mathrm{SM} 2$ & TCR \\
\hline $\mathrm{BP}$ & & 0.341 & 0.4 & 0.438 & 0.35 & 0.188 \\
\hline $\mathrm{HCS}$ & & & 0.286 & 0.347 & 0.341 & 0.161 \\
\hline LTR & & & & 0.467 & 0.327 & 0.165 \\
\hline SM1 & & & & & 0.444 & 0.253 \\
\hline SM2 & & & & & & 0.253 \\
\hline TCR & & & & & & \\
\hline
\end{tabular}

fungi (Hyde \& Goh 1998a). A greater fungal diversity is also found if samples are collected periodically over a longer time period because of the seasonal occurrence of certain taxa. More than 100 fungal taxa were identified from the USA (Shearer \& Crane 1986), from the Tai Po Kau stream, Hong Kong (Ho 1998) and from the LTR Hong Kong (Tsui 1999), which illustrates the need for long-term studies.

With the exception for BP and the SM (October 1998), high values of species richness (ShannonWiener's index), usually of more than 5, were recorded. This is because a large number of species only occurred once or twice. The application of species diversity measures to this study is therefore questionable because the value of indices should not exceed 5 in natural communities (Krebs 1999). Although the number of species may be a better indicator, further studies are required to resolve how well the presence of fruiting bodies on wood reflects the distribution of mycelium inside the wood.

\section{CONCLUSION}

A total of 153 fungal taxa were found in different freshwater streams in Hong Kong, and about 30 species were previously undescribed. These high numbers illustrate the high diversity of fungi in subtropical regions. The fungal community in the TCR was distinct, and there were also variations in species composition between other streams. Differences in riparian vegetation composition, and substrate specificity of fungi are suggested to be important factors in regulating fungal communities. Further research is necessary in order to verify the presence of keystone species, and many species await discovery.

Acknowledgements. We are grateful to Dr T. K. Goh for the identification of mitosporic fungi. We thank 3 anonymous reviewers for constructive comments on an earlier version of this manuscript. Ken Wong, Michelle Wong and Helen Leung are thanked for technical assistance. C.K.M.T. would like to thank The University of Hong Kong for the award of a Postgraduate Studentship.

\section{LITERATURE CITED}

Bärlocher F (1992) The ecology of aquatic hyphomycetes. Springer-Verlag, Berlin

Barr ME (1990) Prodromus to nonlichenized, pyrenomycetous members of class Hymenomycetes. Mycotaxon 39:43-184

Begon M, Harper JL, Townsend CR (1993) Ecology: individuals, population and communities, 3rd edn. Blackwell, Boston

Chan KT (1997) Multivariate analysis of benthic macroinvertebrate communities of Hong Kong streams. MPhil thesis, Department of Ecology and Biodiversity, The University of Hong Kong 
Chauvet E (1991) Aquatic hyphomycete distribution in SouthWestern France. J Biogeogr 18:699-706

Crane JL, Shearer CA, Barr ME (1998) A revision of Boerlagiomyces with notes and a key to the saprobic genera of Tubeufiaceae. Can J Bot 76:602-612

Dudgeon D (1989) The influence of riparian vegetation on the functional organization of four Hong Kong stream communities. Hydrobiologia 179:183-194

Dudgeon D, Corlett R (1994) Hills and streams: an ecology of Hong Kong. Hong Kong University Press, Hong Kong

Fabre E (1996) Relationships between aquatic hyphomycete communities and riparian vegetation in 3 Pyrenean streams. C R Acad Sci Paris, Sci Vie/Life Science 319:107-111

Goh TK, Hyde KD (1996) Biodiversity of freshwater fungi. $\mathrm{J}$ Ind Microbiol 17:328-345

Goh TK, Hyde KD (1999) Fungi on submerged wood and bamboo in the Plover Cove Reservoir, Hong Kong. Fungal Divers 3:57-85

Goh TK. Ho WH, Hyde KD, Tsui KM (1997a) Four new species of Xylomyces (Hyphomycetes) from submerged wood and a key to the genus. Mycol Res 101:1323-1328

Goh TK, Ho WH, Hyde KD, Umali TE (1997b) New records and species of Sporoschisma and Sporoschismopsis from submerged wood in the Tropics. Mycol Res 101:1295-1307

Gönczöl J, Révay A (1993) Further studies on fungal colonization of twigs in the Morgó-stream, Hungary. Nova Hedwig 56:531-542

Gönczöl J, Révay A (1997) Spatial dynamics of fungal populations (hyphomycetes) on submerged corticated twigs. Nova Hedwig 64:553-565

Graça MSA (1993) Patterns and processes in detritus-based stream systems. Limnologica 23:107-114

Harmon ME, Franklin JF, Swanson FJ, Sollins O, Gregory SV, Lattin JD, Anderson NH, Cline SP, Aumen NG, Sedell JR, Lienkaemper GW, Cromack K, Cummins KW (1986) Ecology of coarse woody debris in temperate ecosystems. Adv Ecol Res 15:133-302

Hawksworth DL, Kirk PM, Sutton BC, Pegler DN (1995) Ainsworth \& Bisby's dictionary of the fungi, 8th edn. CAB International, Wallingford

Ho WH (1998) Biodiversity, ecological and ultrastructural studies of freshwater fungi. PhD thesis, Department of Ecology and Biodiversity, The University of Hong Kong

Hoiland K, Bendiksen E (1997) Biodiveristy of wood-inhabiting fungi in a boreal coniferous forest in Sor-Trondelag County, Central Norway. Nord J Bot 16:643-659

Hyde KD, Goh TK (1997) Fungi on submerged wood in a small stream on Mt Lewis, North Queensland Australia. Muelleria 10:145-157

Hyde KD, Goh TK (1998a) Fungi on submerged wood in Lake Barrine, North Queensland, Australia. Mycol Res 102: $739-749$

Hyde KD, Goh TK (1998b) Fungi on submerged wood in the Riviere St Marie-Louis, The Seychelles. S Afr J Bot 64 $330-336$

Hyde KD, Goh TK (1999) Fungi on submerged wood in the River Coln, the Cotswolds, UK. Mycol Res 103:1561-1574

Hyde KD, Wong SW, Jones EBG (1997) Freshwater ascomycetes. In: Hyde $\mathrm{KD}$ (ed) Biodiversity of tropical microfungi. Hong Kong University Press, Hong Kong, p $179-187$

Hyde KD, Goh TK, Steinke TD (1998) Fungi on submerged wood in the Palmiet River, Durban, South Africa. S Afr J Bot 64:151-162

Editorial responsibility: John Dolan,

Edgewater, Maryland, USA
Kent M, Coker P (1992) Vegetation description and analysis A practical approach. Wiley, Boca Raton

Krebs CJ (1999) Ecological methodology, 2nd edn. Benjamin/Cummings, Menlo Park, CA

Magurran AE (1988) Ecological diversity and its measurement. Croom Helm, London

Raviraja NS, Sridhar KR, Bärlocher F (1998) Fungal species richness in Western Ghat streams (southern India): is it related to $\mathrm{pH}$, temperature or altitude? Fungal Divers 1 $179-191$

Rayner ADM, Boddy L (1988) Fungal decomposition of wood. Its biology and ecology. John Wiley \& Sons, Chichester

Révay A, Gönczöl J (1990) Longitudinal distribution and colonization patterns of wood-inhabitating fungi in a mountain stream in Hungary. Nova Hedwig 51:505-520

Shearer CA (1993) The freshwater ascomycetes. Nova Hedwig 56:1-33

Shearer CA (1999) Biogeography of freshwater ascomycetes. In: Book of abstracts. Proc 7th International Marine and Freshwater Mycology Symposium. 1999. City University of Hong Kong, p 5

Shearer CA, Crane JL (1986) Illinois fungi XII. Fungi and myxomycetes from wood and leaves submerged in Southern Illinois swamps. Mycotaxon 25:527-538

Shearer CA, von Bodman S (1983) Pattern of occurrence of ascomycetes associated with decomposing twigs in a midwestern stream. Mycologia 75:518-530

Shearer CA, Webster J (1991) Aquatic hyphomycete communities in the River Teign. IV. Twig colonization. Mycol Res 95:413-420

Shearer CA, Crane JL, Chen W (1999) Freshwater ascomycetes: Ophioceras species. Mycologia 91:145-156

Thomas K, Chilvers GA, Norris RH (1989) Seasonal occurrence of conidia of aquatic hyphomycetes (fungi) in Lees Creek, Australian Capital Territory. Aust J Mar Freshw Res 40:11-23

Thomas K, Chilvers GA, Norris RH (1992) Aquatic hyphomycetes from different substrates: substrate preference and seasonal occurrence. Aust J Mar Freshw Res 43:491-509

Triska FJ, Cromack K $(1980)$ The role of wood debris in forests and streams. In: Waring $\mathrm{RH}$ (ed) Forest: fresh perspectives from ecosystem analysis. Proc 40th Biology Colloquium, 1979. Oregon University Press, Corvallis, p $171-190$

Tsui KM (1999) Biodiversity and longitudinal distribution of fungi on submerged wood, with reference to human disturbance. PhD thesis, Department of Ecology and Biodiversity, The University of Hong Kong

Vannote RL, Minshall GW. Cummins KW, Sedell JR, Cushing CE (1980) The river continuum concept. Can J Fish Aquat Sci 37:130-137

Wong MKM, Goh TK, Hodgkiss IJ, Hyde KD, Ranghoo VM Tsui CKM, Ho WH, Wong WSW, Yuen TK (1998) Role of fungi in freshwater ecosystem. Biodivers Conserv 7: $1187-1206$

Wong SW, Hyde KD, Jones EBG (1998) Annulatascaceae, a new ascomycete family from the tropics. Syst Ascomyce. tum 16:17-25

Wood-Eggenschwiler S, Bärlocher F (1985) Geographical distribution of Ingoldian fungi. Verh Int Ver Theor Angew Limnol 22:2780-2785

Yuen TK, Hyde KD, Hodgkiss IJ (1999) Physiological growth parameters and enzyme production in tropical freshwater fungi. Mater Org 32:1-16

Submitted: November 2, 1999; Accepted: February 22, 2000

Proofs received from author(s): April 17, 2000 